\title{
A Life at Sea and the Corrosion Fatigue Lives of Offshore Structures
}

\author{
Daniel J. Thomas
}

Submitted: 10 December 2020/in revised form: 26 January 2021/Accepted: 15 April 2021/Published online: 25 April 2021

(C) ASM International 2021

Offshore structures are typically at the front end of service for decades. Undergoing unsymmetrical loading forces, harsh marine conditions, freezing cold temperatures and importantly corrosion. During this time, even a well-designed and optimized structure can undergo structural failure in some of the harshest and most unforgiving environments on earth. This is particularly the case with that of oil platforms as shown in Fig. 1a. These steel structures are subjected to extensive cyclical loading during their service out at sea, this can be one of the main causes structural of failure [1]. As of 2020 there are 1470 offshore oil rigs, with the majority working in the Gulf of Mexico, the North Sea and in the Persian Gulf. Each of which is involved with the skilled work of extracting crude oil wealth from vast reserves beneath the ocean. These harsh marine environments and the presence of corrosion can also accelerate fatigue failure of a structure. This occurs due to electrochemical reactions and plastic deformation in the steel structures that accelerate anodic dissolution of metals and this results in corrosion fatigue crack initiation. The resulting effect on such structures can be a catastrophic failure.

This recently has led me to examine the corrosion fatigue durability behavior of S355 steel, which is used in offshore structural applications. A key failure mechanism for corrosion fatigue is that cracks initiate at stress raising pits [2]. Corrosion pits are formed at the surface of steel structures during service, and these can result in the initiation of fatigue cracks. To test the catastrophic effect of corrosion on structural steel grades, the performance was

D. J. Thomas $(\bowtie)$

Creation Engineering Group, Llynfi Enterprise Centre, Bridgend, UK

e-mail: daniel.thomas@creationengineering.co.uk compared to parent steel structures. In the field, this was carried out by attaching sample specimens to the outer hull of an offshore structure so that they could be exposed to the elements for predetermined periods of time.

It was surprising to see the damage caused, even after a relatively short period time. As shown in Fig. 1b, within $50 \mathrm{~h}$, hard iron oxide had formed with further deterioration and the formation of salt crystals had become extensive. These electrochemical processes cause in the first instance cosmetic damage, but beyond this they also create microsized pits in the structure which ultimately result in localized rises in stress. Strain induced across the component, together with any formed corrosion pits promotes dissolution of the matrix and subsequently results in further pit growth. It is this that can be the starting point for the complete structural failure of an application.

To test the effects of corrosion on the lives of a structure, laboratory-based tests were then carried out on retrieved test specimens. These data can then be used to understand the significant loss of fatigue lives. Since plastic strain has significant effect on the anodic activity of steels, four-point bend testing was carried out on samples as shown in Fig. 2.

It was staggering to observe the significant loss of fatigue life of corroded specimens, even after a relatively short period of time. During corrosion fatigue testing, pits continue to grow into the material. As a pit reaches a critical depth, then a fatigue crack will develop and propagate throughout the structure. With $1000 \mathrm{~h}$ of exposure to marine conditions, at the lower stress levels (high cycle fatigue), specimens lasted fifty times less than that of the fatigue life of uncorroded specimens. After later sectioning and analysis, there were observed too many hundreds of pits that were photographed transverse to the surface of the specimens. 
Fig. 1 (a) Extensive corrosion observed on an offshore oil platform and (b) Microscope images taken in the field of S355 steel after it was left for periods of time when exposed to the harsh marine environment. Within $500 \mathrm{~h}$ it was observed that excessive salt crystals and iron oxide had grown across the surface
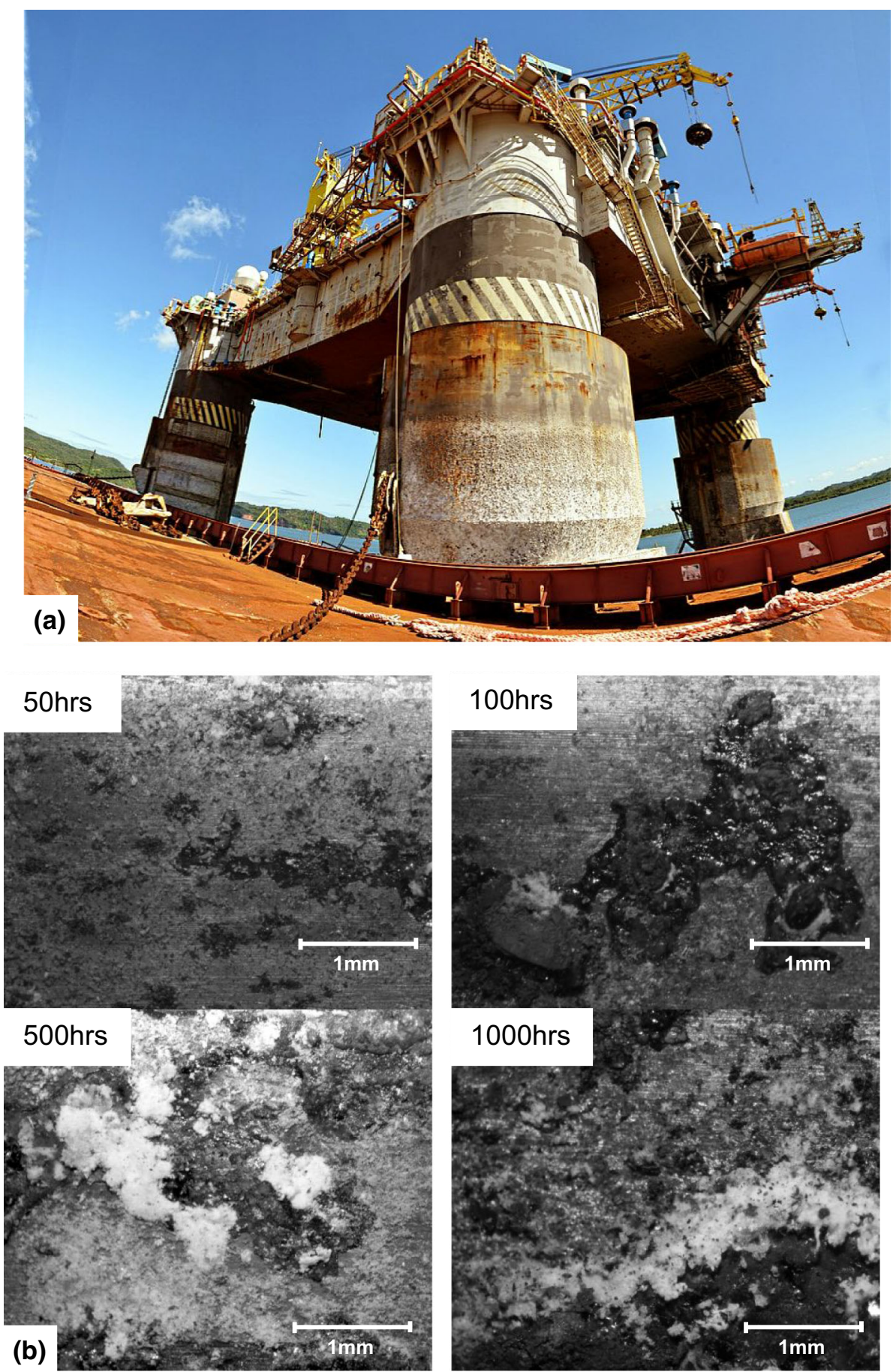

There are two critical mechanisms that effect the behavior of corrosion fatigue crack propagation in marine environments. These are hydrogen embrittlement and anodic dissolution at the crack tip. As the length of a crack increases as shown in Fig. 3, oxygen from the surface has further to diffuse to the crack tip. As a result, this leads to a reduction in the oxygen concentration at the crack tip. Further to this, hydrogen embrittlement results in an 


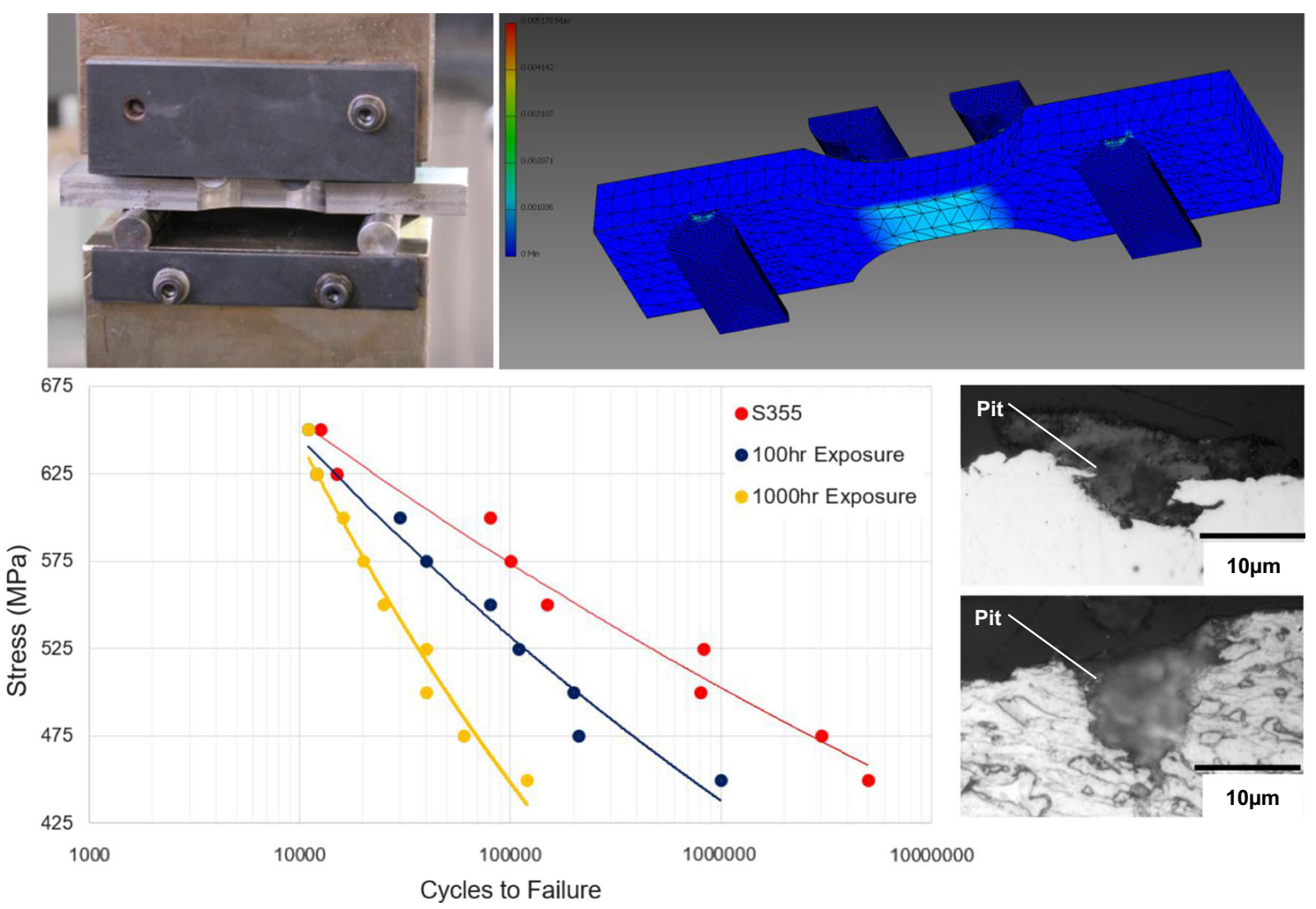

Fig. 2 Test samples of S355 steel under four-point laboratory fatigue testing. The resulting stress analysis and fatigue life curves to the point of specimen failure showing the significant reduction in life due to exposure to marine conditions. Micrographs of two stress raising pits generated of eth steel surface after $1000 \mathrm{~h}$ of exposure. acceleration in crack growth rate. This is dependent on the intensity of the stress and the induced cyclical frequency. Subsequent crack growth rate due to hydrogen embrittlement occurs when a critical stress intensity level is achieved. Under the process of sinusoidal fatigue loading, the orientation properties of the crack were observed to change. As a result, there is a transition from predominantly transgranular crack growth to that of intergranular and secondary crack growth resulting in rapid failure of the application [3].
It is often wise to be mindful that even the largest structures are vulnerable to the smallest defects. During subsequent testing, it was clear to observe the slow growth in the region I crack, followed by a rapid region II crack growth. It is this factor that leads to sudden and catastrophic failures of heavy industrial structures. It is the fact that exposed metal surfaces are critical towards the initiation of pits and the growth of a fatigue crack that determine the life of a structure. Therefore, it is important that offshore structures do not deteriorate to the level at which defects become observed and that structures are 
Fig. 3 Microstructure of a fatigue crack through S355 (etched with $2 \% \mathrm{Nital}$ ) and the change in crack orientation showing the growth of a corrosion induced fatigue crack during the loading process and crack growth range

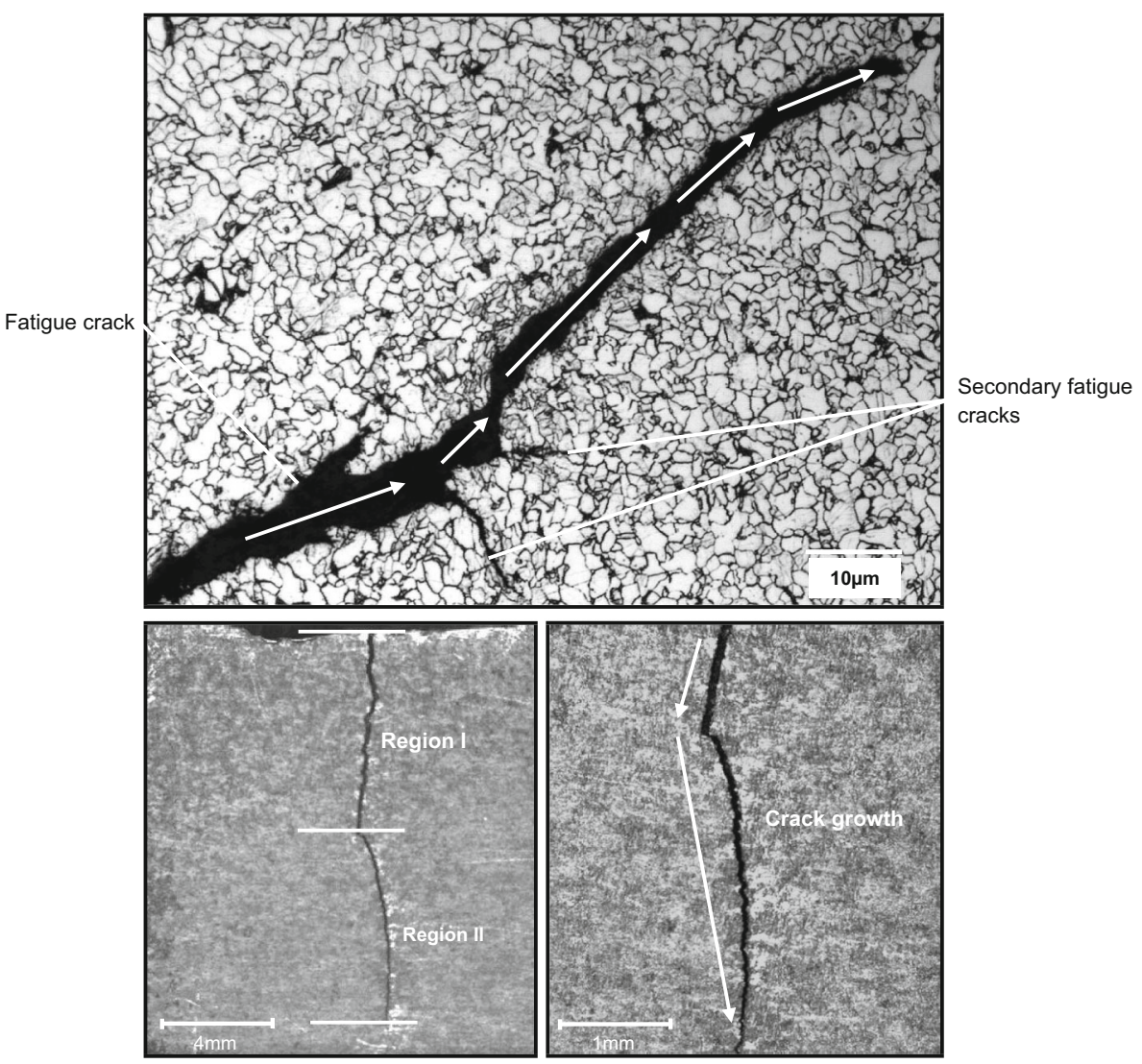

continuously well maintained. This preventative strategy ultimately results significantly less costly problems in the long run.

\section{References}

1. M. Jakubowski, Fatigue crack propagation in austenitic stainless steel under low frequency loading and saltwater conditions. Fatigue Fract. Eng. Mater. Struct. 21, 937-946 (1998)
2. B. Tomkins, Role of mechanics in corrosion fatigue. Met. Sci. 13, 387-395 (1979)

3. S. Suresh, R.O. Ritchie, Mechanistic dissimilarities between environmentally influenced fatigue crack propagation at near threshold and higher growth rates in lower strength steels. Met. Sci. 16, 529-538 (1982)

Publisher's Note Springer Nature remains neutral with regard to jurisdictional claims in published maps and institutional affiliations. 